\title{
Asymmetries of the retinal nerve fibre layer thickness in normal eyes
}

Shinshu University School of Medicine, Department of Ophthalmology, Matsumoto 390-8621, Japan

Y Kurimoto

K Matsuno

Y Kaneko

J Umihira

N Yoshimura

Correspondence to: Yasuo Kurimoto, Department of Ophthalmology, Shinshu University School of Medicine, 3-1-1 Asahi, Matsumoto, 390-8621, Japan kurina@

hsp.md.shinshu-u.ac.jp

Accepted for publication 13 January 2000

\author{
Yasuo Kurimoto, Kaori Matsuno, Yumi Kaneko, Junichi Umihira, Nagahisa Yoshimura
}

\begin{abstract}
Aims-To investigate the variation in the retinal nerve fibre layer thickness in detail in normal eyes with a scanning laser polarimeter.

Methods-The retinal nerve fibre layer thickness (RNFLT) was measured in 94 normal volunteers with a scanning laser polarimeter. The mean RNFLT around a 10 pixel-wide ellipse located concentrically with the disc of 1.5 disc diameters was calculated for 16 sectors each of 22.5 degrees. The symmetry of the RNFLT distribution with respect to the horizontal midline for individual eyes and to the vertical meridian for the two eyes was examined.

Results-The RNFLT was thicker on the inferior side than on the superior side for the temporal four pairs of 22.5 degrees sectors, and the differences were significant in two of the four temporal pairs $(p<0.007)$. The RNFLT was thicker in the superior than in the inferior side for the nasal four pairs of the sectors, and the differences were significant in three of the four nasal pairs $(p<0.04)$. The mean RNFLT was significantly thicker in the right eyes than in the left eyes in the four temporal sectors $(p<0.02)$, and significantly thicker in the left eyes than in the right eyes in the inferior two nasal sectors $(\mathbf{p}<0.01)$.

Conclusions-Asymmetries of the RNFLT in normal eyes with respect to the horizontal midline and to the vertical meridian for the two eyes were found. These asymmetries should be considered when retinal nerve fibre layer loss is evaluated during the course of a disease process. (Br f Ophthalmol 2000;84:469-472)
\end{abstract}

The nerve fibre analyser (NFA), first described by Weinreb and colleagues in $1990,{ }^{12}$ is a scanning laser polarimeter designed for making quantitative measurements of the retinal nerve fibre layer thickness (RNFLT) in humans. A study in monkeys showed a good correlation between the value of RNFLT as determined with the NFA and the histopathological measurement. ${ }^{1}$ In several clinical studies on humans, high reproducibility of measurements obtained with the NFA was reported, ${ }^{2-4}$ and the measurements were also reported to correspond to known properties of the retinal nerve fibre layer in normal and glaucomatous eyes. ${ }^{2}$ Before the development of the NFA, the quantitative evaluation of the retinal nerve fibre layer loss was difficult and tended to lack objectivity and precision. After the development of the NFA, it became possible to measure the RNFLT quantitatively with good reproducibility. ${ }^{2-4}$

A number of studies that examined the retinal nerve fibre layer loss using the NFA have been published. ${ }^{25-11}$ However, there are some inconsistencies in the reports on the distribution of RNFLT in normal eyes, and the RNFLT in normal eyes has not been investigated in detail with the NFA. For instance, there is a question whether RNFLT is symmetrical with respect to the horizontal midline or not. Some studies have reported that the RNFLT in normal eyes was different in the superior and inferior sectors, ${ }^{28}$ while another study stated that the RNFLT was symmetrical with respect to the horizontal midline regardless of age. ${ }^{6}$ In fact, previous studies could not answer such a fundamental question concerning the normal distribution of RNFLT. Thus, a more detailed investigation of the distribution of the RNFLT in normal eyes is necessary in order to know if a retinal nerve fibre layer loss has actually occurred.

In most of the previous studies using the NFA, the RNFL was broadly divided into two or four sectors in a peripapillary ellipse around the optic disc, ${ }^{235-79}$ and only the mean RNFLT in such a broad area was used for the analysis. To investigate the RNFLT in normal eyes in more detail, we divided the peripapillary ellipse into 16 equal sectors and examined the variation of the RNFLT with respect to the horizontal midline for individual eyes and to the vertical meridian for the two eyes.

\section{Materials and methods}

There were 94 normal Japanese volunteers (53 men, 41 women: mean age 37.4 (SD 15.9) years, range 11-72 years) enrolled in the study. None of the subjects had any ocular or systemic diseases (such as diabetes mellitus) that could alter RNFLT. To assess the difference between the superior and inferior RNFLT, one eye was randomly selected from each volunteer for analysis (47 right eyes and 47 left eyes). To assess the difference between the right and left eyes, the 124 eyes of 62 volunteers whose two eyes were examined were enrolled. All procedures were conducted in accordance with the principles embodied in the Declaration of Helsinki, and written informed consent was obtained from all subjects.

The RNFLT was measured with the NFA-II scanning laser polarimeter (Laser Diagnostic Technologies, San Diego, CA, USA). The technique exploits the birefringent properties 
Table 1 Age, refraction, and axial length of the subjects (mean (SD))

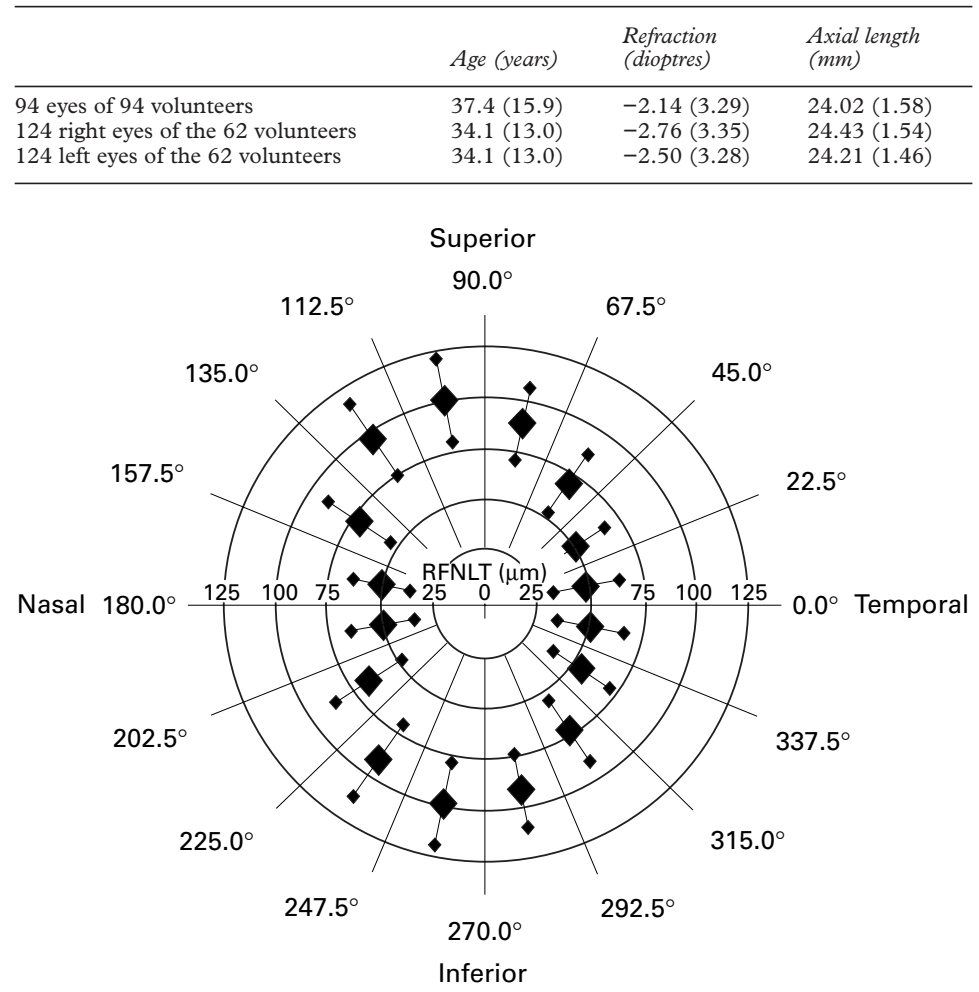

Figure 1 The mean RNFLT of the 16 sectors for the 94 eyes of the 94 volunteers. Data are plotted in polarographic coordinates. Error bars represent the standard deviations.

of the retinal nerve fibre layer. The light source of this instrument consists of a near infrared diode laser (wavelength $780 \mathrm{~nm}$ ) whose state of polarisation is modulated. The polarised light penetrates the birefringent nerve fibre layer and is partially reflected from the deeper layers of the retina. The polarisation detection unit analyses the state of polarisation of the reflected light. A scanning unit deflects the laser beam to adjacent $256 \times 256$ retinal positions, and at each position, the computer algorithm calculates the change in the polarisation state as the amount of retardation and expresses it as the RNFLT. In the map of 256 $\times 256$ pixels, the RNFLT for each position is determined.

In the present study, the RNFLT was measured along a 10 pixel-wide ellipse that was concentric with the optic disc and was 1.5 disc diameter. The mean RNFLT was calculated for the entire peripapillary retina $(0-360$ degrees), for the superior and inferior halves (0-180 degrees, and 180-360 degrees), for four 90 degree quadrants (superior 45-135 degrees, nasal 135-225 degrees, inferior 225315 degrees, and temporal 315-45 degrees), and for 16 equal 22.5 degrees sectors $(0-22.5$, 22.5-45, 45-67.5, 67.5-90, 90-112.5, 112.5$135, \quad 135-157.5, \quad 157.5-180, \quad 180-202.5$, $202.5-225, \quad 225-247.5, \quad 247.5-270, \quad 270$

Table 2 Differences between the superior and inferior sectors (mean (SD))

\begin{tabular}{lllll}
\hline Division & Superior & Inferior & p Value & $\begin{array}{l}\text { 95\% CI of the } \\
\text { difference }\end{array}$ \\
\hline $0-180$ v 180-360 degrees & $72.67(14.80)$ & $71.81(14.80)$ & 0.181 & -0.41 to 2.13 \\
$45-135$ v 225-315 degrees & $90.03(17.16)$ & $89.24(16.97)$ & 0.420 & -1.14 to 2.72 \\
\hline
\end{tabular}

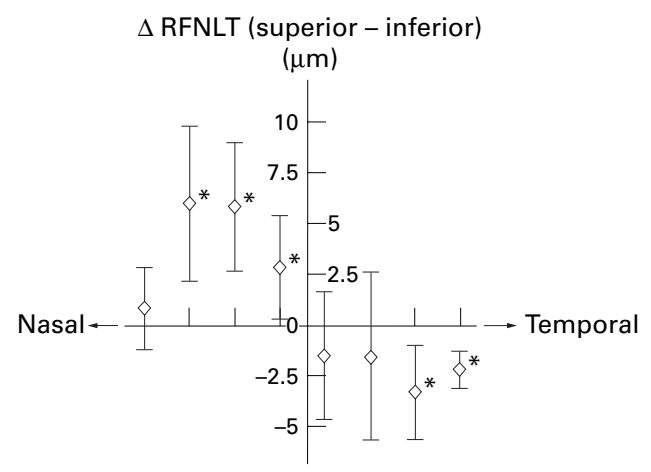

Figure 2 The mean difference between the superior eight sectors of 22.5 degrees and the inferior homotopical sectors. The RNFL was thicker in the inferior than the superior halves for the temporal four pairs of the sectors, and the differences were statistically significant in two of the four pairs $\left({ }^{*} p<0.0001,95 \%\right.$ confidence interval: -3.08 to -1.28 for the most temporal pairs and $p=0.006,95 \%$ confidence interval: -5.63 to -0.97 for the next temporal pairs). The RNFL was thicker in the superior sides than in the inferior sides for the nasal four pairs of sectors, and the differences were statistically significant in three of the four pairs $\left({ }^{*} p=0.0025,95 \%\right.$ confidence interval: 2.17 to 9.81 for the second nasal pairs, $p=0.0005,95 \%$ confidence interval: 2.64 to 9.00 for the third nasal pairs, and $p=$ $0.031,95 \%$ confidence interval: 0.27 to 5.39 for the fourth nasal pairs). Error bars represent the $95 \%$ confidence intervals.

292.5, 292.5-315, 315-337.5, and 337.5-360 degrees). The final results represent the mean of the measurements obtained from three consecutive images.

The refraction and the axial length of all subjects were measured with a Nidek ARK900 autorefractometer (Nidek, Tokyo, Japan) and a Nidek EchoScan US-900 (Nidek), respectively.

The Student's two tailed paired $t$ test was used to determine whether differences between the RNFLT in the superior and inferior sectors were significant, and the correlations between the differences of RNFLT and the age, refraction, and axial length was assessed by determining the Pearson's correlation coefficients from which statistical significance was evaluated by using Fisher's $\mathrm{Z}$ transformation. Similarly, differences between the RNFLT of the right and left eyes were analysed by using the Student's two tailed paired $t$ test, and the correlations between the differences of RNFLT and the age or the differences of refraction and axial length between both eyes were assessed by determining the Pearson's correlation coefficients from which statistical significance was evaluated by using the Fisher's $\mathrm{Z}$ transformation. The data are expressed as mean (SD), and a p value of less than 0.05 was accepted as statistically significant.

\section{Results}

The means (SD) of the age, refraction, and axial length of the 94 eyes of the 94 volunteers, and those of the 124 eyes of 62 volunteers are shown in Table 1 . The mean RNFLT of the 16 sectors in the 94 eyes are plotted in polar coordinates in Figure 1. In agreement with previous reports, the RNFL was thicker in the superior and inferior retina than in the nasal and temporal retina. 
Table 3 Differences between the right and left eyes (mean (SD))

\begin{tabular}{lllll}
\hline Sector & Right & Left & p Value & $\begin{array}{l}\text { 95\% CI of the } \\
\text { difference }\end{array}$ \\
\hline Overall peripapillary: 0-360 degrees & $73.59(13.04)$ & $71.67(14.01)$ & 0.431 & -2.89 to 6.74 \\
$\quad$ superior half: 0-180 degrees & $72.88(13.82)$ & $70.18(13.61)$ & 0.276 & -2.18 to 7.57 \\
$\quad$ inferior half: $180-360$ degrees & $71.86(13.31)$ & $70.83(14.45)$ & 0.681 & -3.91 to 5.97 \\
Temporal quadrant: 315-45 degrees* & $54.14(13.72)$ & $46.13(12.79)$ & 0.001 & 3.29 to 12.73 \\
Superior quadrant: $45-135$ degrees & $89.92(16.34)$ & $88.60(16.80)$ & 0.658 & -4.57 to 7.21 \\
Nasal quadrant: $135-225$ degrees & $57.69(12.47)$ & $61.20(14.92)$ & 0.159 & -8.39 to 1.39 \\
Inferior quadrant: 225-315 degrees & $90.06(14.61)$ & $87.60(17.30)$ & 0.390 & -3.23 to 8.15 \\
\hline
\end{tabular}

DIFFERENCE OF RNFLT BETWEEN THE SUPERIOR AND INFERIOR SIDES

When the peripapillary ellipse was divided into the superior and inferior halves or into the four quadrants in the conventional way, no significant difference in the RNFLT was found between the superior and inferior parts (Table 2). However, when the analysis was done for the eight individual sectors in the superior and inferior halves, significant differences were found. The differences (RNFLT of the superior minus the RNFLT in the inferior sector) between the superior eight sectors and the inferior homotopical sectors and the $95 \%$ confidence intervals are plotted in Figure 2. The RNFLT was thicker in the inferior than in the superior side for the temporal four pairs of sectors and the differences were statistically significant in two of the four temporal pairs ( $p$ $<0.007)$. On the other hand, the RNFLT was thicker in the superior than in the inferior sectors for the nasal four pairs of sectors, and the differences were significant in three of the four nasal pairs $(\mathrm{p}<0.04)$.

No significant correlation was found between the superior-inferior differences of RNFLT and the age, refraction, and axial length.

DIFFERENCES OF THE RNFLT BETWEEN THE RIGHT AND LEFT EYES

The differences of RNFLT between the right and left eyes for the superior and inferior half of the ellipse and for the four conventional quadrants are shown in Table 3. The RNFLT

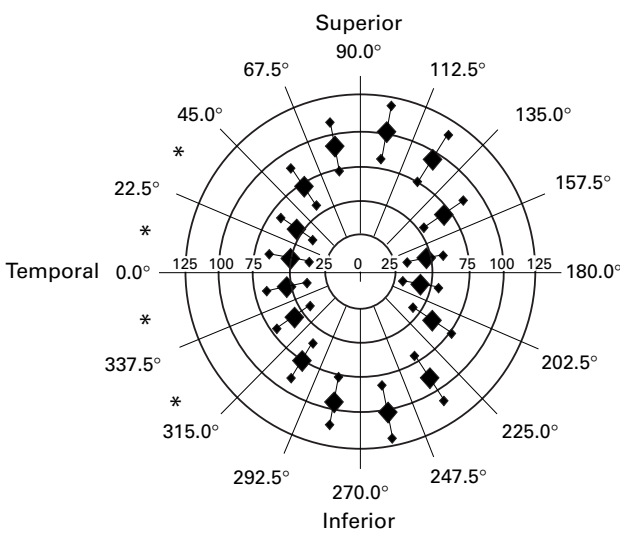

Right eyes was thicker in the right eyes than in the left eyes in the temporal quadrants and the difference was statistically significant $(p=0.001)$.

The mean RNFLT of each of the 16 sectors of 22.5 degrees for the right and left eyes are shown in Figure 3. In the temporal four sectors (315-337.5, 337.5-360, 0-22.5, and 22.5-45 degrees), the mean RNFLT was significantly thicker in the right eyes than in the left eyes $(p$ $<0.02)$. In the two inferior-nasal sectors (180202.5 and 202.5-225 degrees), the mean RNFT was significantly thicker in the left eyes than in the right eyes $(\mathrm{p}<0.001)$.

No significant correlation was found between the right-left differences of RNFLT and the age, differences of the refraction, and axial length between the two eyes.

\section{Discussion}

In previous reports that examined normal eyes with the NFA, the RNFLT was analysed by dividing the peripapillary ellipse into only two or four broad parts, nor was the precise distribution studied in detail. ${ }^{236} \mathrm{In}$ addition, the main aim of those studies was to examine whether the results obtained with the NFA were in good agreement with the conventional findings of RNFLT and did not present any new findings on the RNFLT obtained by the NFA.

There is a controversy whether RNFLT is symmetrical with respect to the horizontal midline. ${ }^{268}$ The present study demonstrated that the distribution of RNFLT is not symmetrical and the RNFLT is thicker on the inferior side of the temporal retina and thicker on the superior side of the nasal retina. In the earlier reports, the analysed sectors to compare the difference between the superior and inferior sides included equally both temporal and nasal portions. ${ }^{6}$ Therefore, the difference between the superior and inferior sides, found in our study, was not detected because the differences between the temporal and nasal portions were cancelled out.

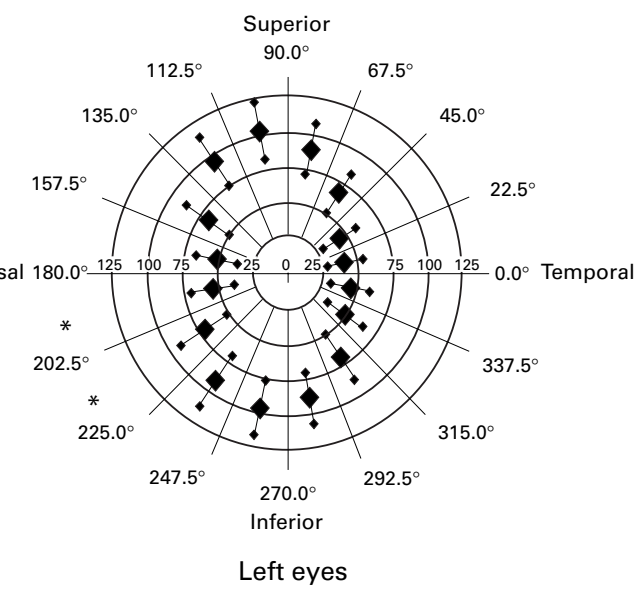

Figure 3 The mean RNFLT of 16 sectors of 22.5 degrees for the right and left eyes are plotted in polarographic coordinates. The mean RNFL was significantly thicker in the right eyes than in the left eyes for the temporal four sectors $\left({ }^{*} p=0.0004,95 \%\right.$ confidence interval: 4.23 to 14.29 for $0-22.5$ degrees, $p=0.0005$, 95\% confidence interval: 4.12 to 13.94 for 22.5-45 degrees, $p=0.020$, 95\% confidence interval: 0.99 to 11.46 for $315-337.5$ degrees, and $p=0.0077$, 95\% confidence interval: 1.91 to 12.25 for 337.5-360 degrees), and significantly thicker in the left eyes than in the right eyes for the inferior-nasal two sectors ${ }^{*} p=0.0005$, 95\% confidence interval: -14.33 to -4.12 for $180-202.5$ degrees and $p=0.0098,95 \%$ confidence interval: -15.08 to -2.11 for 202.5-225 degrees). Error bars represent the standard deviations. 
As far as we know, there has been no previous report about the asymmetries in the RNFLT between the right and left eyes as found in this study. We found that the RNFLT was significantly thicker in the right than in the left eyes for the temporal quadrant (315-45 degrees) and thicker in the left eyes than in the right eyes for an inferior-nasal sector (180-225 degrees).

Thus, the RNFLT in normal eyes is not symmetrical in either the superior-inferior axis or the right-left axis. In general, humans do show various asymmetries in visual function between the superior and inferior sides, and between the right and left eyes. For example, it is known that the threshold perimetric values are higher in the inferior hemifield than in the superior one, ${ }^{12}$ and a histomorphometric study showed that the RNFLT was thicker at the inferior disc border than at the superior disc margin. ${ }^{13}$ On the other hand, another histological study showed that the density of retinal ganglion cells was higher in the superior than in the inferior retina. ${ }^{14}$ Thus, the asymmetries of the RNFLT between the superior and inferior retina should not be too surprising. However, the reason why there should be a difference in the RNFLT between the two eyes and why the difference in the superior and inferior sides is reversed for the temporal and the nasal sides is puzzling. However, a difference of retinal ganglion cells density between right and left eyes has been reported for the human retina. ${ }^{13}$ The present results suggest that these asymmetries in the RNFLT are not caused by ageing and are not affected by the refraction or axial length, because no significant correlation was found between the differences of RNFLT and the age, refraction, and axial length. Further investigations will be necessary to determine the physiological significance of these asymmetries of RNFLT.

In summary, the present study demonstrated that the RNFLT in normal eyes is not symmetrical with respect to the horizontal midline for individual eyes and not symmetrical to the vertical meridian for the two eyes. These findings must be considered when a retinal nerve fibre layer loss is believed to have occurred during the course of a disease process.

1 Weinreb RN, Dreher AW, Coleman A, et al. Histopathologic validation of Fourier-elipsometry measurements of retinal nerve fiber layer thickness. Arch Ophthalmol 1990;108:55760.

2 Weinreb RN, Shakiba S, Zangwill L. Scanning laser polarimetry to measure the nerve fiber layer of normal and polarimetry to measure the nerve fiber layer of normal an

3 Qi-Min C, Tomita G, Inazumi K, et al. Evaluation of the effect of aging on the retinal nerve fiber layer thickness using scanning laser polarimetry. F Glaucoma 1995;4:40613.

4 Zangwill L, Berry CA, Garden VS, et al. Reproducibility of retardation measurements with the nerve fiber analyzer II. f Glaucoma 1997;6:384-9.

5 Weinreb RN, Shakiba S, Sample PA, et al. Association between quantitative nerve fiber layer measurement and visual field loss in glaucoma. Am f Ophthalmol 1995;120: $732-8$.

6 Tjon-Fo-Sang MJ, Vries J, Lemij HG. Measurement by nerve fiber analyzer of retinal nerve fiber layer thickness in normal subjects and patients with ocular hypertension. Am 7 Ophthalmol 1996;122:220-7.

7 Tjon-Fo-Sang MJ, Lemij HG. The sensitivity and specificity of nerve fiber layer measurements in glaucoma as of nerve fiber layer measurements in glaucoma as mol 1997;123:62-9.

8 Anton A, Zangwill L, Emdadi A, et al. Nerve fiber layer measurements with scanning laser poloarimetry in ocular hypertension. Arch Ophthalmol 1997;115:331-4.

9 Hollo G, Suveges I, Nagymihaly A, et al. Scanning laser polarimetry of the retinal nerve fibre layer in primary open angle and capsular glaucoma. Br f Ophthalmol 1997;81: 857-61.

10 Choplin NT, Lundy DC, Dreher AW. Differentiating patients with glaucoma from glaucoma suspects and normal subjects by nerve fiber layer assessment with scanning laser polarimetry. Ophthalmology 1998;105:2068-76.

11 Steel DH, Waldock A. Measurement of the retinal nerve fibre layer with scanning laser polarimetry in patients with previous demyelinating optic neuritis. 7 Neurol Neurosurg Psychiatry 1998;64:505-9.

12 Harrington DO, Drake MV. Normal visual field. In: Harrington DO, Drake MV, eds. The visual fields. 6 th ed. St Louis: Mosby, 1990:93-103.

13 Dichtl A, Jonas JB, Naumann GO. Retinal nerve fiber layer thickness in human eyes. Graefes Arch Clin Exp Ophthalmol $1999 ; 237: 474-9$.

14 Curcio CA, Allen KA. Topography of ganglion cells in human retina. $\mathcal{F}$ Comp Neurol 1990;300:5-25. 